\title{
Publisher Correction: An indoor rice model
}

Jun Lyu

Correction to: Nature Plants https://doi.org/10.1038/s41477-018-0284-9, published online 4 October 2018.

In the version of this Research Highlight originally published, the linked paper was referenced as published in Plant Cell, the correct journal is Molecular Plant. This citation has now been amended. 